polynomials on a finite interval of the real axis]. Izvestiia AN SSSR. Ser. matematicheskaia, 1958, vol. 22, no. 3, pp. 337-354 (in Russian).

7. Freud G. Über die Approximation Reelen Stetiger Functionen Durch Gewohnliche Polinome. Math. Ann., 1959, vol. 137, no. 1, pp. 17-25.

8. Teljakovskii S. A. Two theorems on approximation of functions by algebraic polynomials. Mat. Sb. (N.S.), 1966, vol. 70(112), no. 2, pp. 252-265 (in Russian).

9. Brudnyi Yu. A. The approximation of functions by algebraic polynomials. Mathematics of the USSRIzvestiya, 1968, vol. 2, no. 4, pp. 735-743. DOI: 10.1070/IM1968v002n04ABEH000662

10. Lorentz G. G., Zeller K. L. Degree of Approximation by Monotone Polynomials. II. J. Approx. Theory, 1969, vol. 2, no. 3, pp. 265-269.

11. Shevchuk I. A. Priblizhenie mnogochlenami $i$ sledy nepreryonykh na otrezke funktsii [Approximation by polynomials and traces continuous on the interval functions]. Kiev, Naukova dumka, 1992. 225 p. (in Russian)

12. Shvedov A. S. Jackson's theorem in $L^{p}, 0<p<1$, for algebraic polynomials, and orders of comonotone approximations. Math. Notes, 1979, vol. 25, no. 1, pp. 5763.

13. Shvedov A. S. Komonotonnoe priblizhenie funktsii mnogochlenami [Comonotone approximation of functions by polynomials]. Dokl. Akad. Nauk SSSR. 1980, vol. 250, no. 1, pp. 39-42 (in Russian).

14. Dzyadyk V. K. Vvedenie v teoriiu ravnomernogo priblizheniia funktsii polinomami [Introduction to the theory of uniform approximation of functions by polynomials]. Moscow, Nauka, 1977, 512 p. (in Russian)

УДК 512.572

\title{
О ТОЖДЕСТВАХ СПЕЦИАЛЬНОГО ВИДА В АЛГЕБРАХ ПУАССОНА
}

\section{С. М. Рацеев}

Кандидат физико-математических наук, доцент кафредры инсрормационной безопасности и теории управления, Ульяновский государственный университет, RatseevSM@mail.ru

В работе рассматриваются так называемые customary и extended customary тождества в алгебрах Пуассона. Показано, что последовательность коразмерностей $\left\{r_{n}(V)\right\}_{n \geq 1}$ любого extended customary пространства многообразия алгебр Пуассона $V$ над произвольным полем либо ограничена полиномом, либо не ниже показательной срункции с основанием степени, равной 2. При этом если данная последовательность ограничена полиномом, то найдется такой многочлен $R(x)$ с рациональными коэсрсициентами, что $r_{n}(V)=R(n)$ для всех достаточно больших $n$. Приводится нижняя и верхняя границы для многочленов $R(x)$ произвольной фриксированной степени.

Ключевые слова: алгебра Пуассона, многообразие алгебр, рост многообразия.

Векторное пространство $A$ над полем $K$ с двумя $K$-биллинейными операциями умножения «»» и «\{,\}» называется алгеброй Пуассона, если относительно операции «•» пространство $A$ является коммутативной ассоциативной алгеброй с единицей, относительно операции «\{, $\} »-$ алгеброй Ли, и данные операции связаны правилом Лейбница:

$$
\{a \cdot b, c\}=a \cdot\{b, c\}+\{a, c\} \cdot b, \quad a, b, c \in A .
$$

Алгебры Пуассона возникают в различных разделах алгебры, дифференциальной геометрии, топологии, современной теоретической физики и т. д.

Пусть $F(X)$ - свободная алгебра Пуассона, где $X=\left\{x_{1}, x_{2}, \ldots\right\}$ - счетное множество свободных образующих. Обозначим через $P_{n}$ пространство в $F(X)$, состоящее из полилинейных элементов степени $n$ от переменных $x_{1}, \ldots, x_{n}$.

Выделим в пространстве $P_{2 n}$ подпространство $Q_{2 n}$, порожденное элементами вида

$$
\left\{x_{a_{1}}, x_{a_{2}}\right\} \cdot\left\{x_{a_{3}}, x_{a_{4}}\right\} \cdot \ldots \cdot\left\{x_{a_{2 n-1}}, x_{a_{2 n}}\right\} .
$$

Тогда данное пространство есть линейная оболочка следующих элементов:

$$
\begin{gathered}
Q_{2 n}=\left\langle\left\{x_{\tau(1)}, x_{\tau(2)}\right\} \cdot\left\{x_{\tau(3)}, x_{\tau(4)}\right\} \cdot \ldots \cdot\left\{x_{\tau(2 n-1)}, x_{\tau(2 n)}\right\}\right| \tau \in S_{2 n}, \\
\tau(1)<\tau(2), \tau(3)<\tau(4), \ldots, \tau(2 n-1)<\tau(2 n), \\
\tau(1)<\tau(3)<\ldots<\tau(2 n-1)\rangle_{K} .
\end{gathered}
$$


Обозначим через $T_{2 n}$ множество перестановок $\tau$ из $S_{2 n}$, которые удовлетворяют указанным выше свойствам. Пространство $Q_{2 n}$ было введено Д. Фаркашом (D. R. Farkas) в работах [1,2]. Важность рассмотрения данных пространств показывает следующая теорема.

Теорема 1 [1]. Пусть V - многообразие алгебр Пуассона над полем нулевой характеристики, в котором выполнено нетривиальное тождество. Тогда в $\mathbf{V}$ выполняется нетривиальное тождество вида

$$
\sum_{\tau \in T_{2 n}} \alpha_{\tau}\left\{x_{\tau(1)}, x_{\tau(2)}\right\} \cdot\left\{x_{\tau(3)}, x_{\tau(4)}\right\} \cdot \ldots \cdot\left\{x_{\tau(2 n-1)}, x_{\tau(2 n)}\right\}=0, \quad \alpha_{\tau} \in K .
$$

Определим также подпространство $R_{n}$ в $P_{n}$, порожденное элементами вида

$$
\left\{x_{a_{1}}, x_{a_{2}}\right\} \cdot\left\{x_{a_{3}}, x_{a_{4}}\right\} \cdot \ldots \cdot\left\{x_{a_{2 m-1}}, x_{a_{2 m}}\right\} \cdot x_{\alpha_{2 m+1}} \cdot \ldots \cdot x_{\alpha_{n}} .
$$

Тогда пространство $R_{n}$ является линейной оболочкой элементов следующего вида:

$$
\begin{gathered}
R_{n}=\left\langle\left\{x_{\tau(1)}, x_{\tau(2)}\right\} \cdot\left\{x_{\tau(3)}, x_{\tau(4)}\right\} \cdot \ldots \cdot\left\{x_{\tau(2 m-1)}, x_{\tau(2 m)}\right\} \cdot x_{\tau(2 m+1)} \cdot \ldots \cdot x_{\tau(n)}\right|, \\
\tau \in S_{n}, \quad 0 \leq 2 m \leq n \\
\tau(1)<\tau(2), \tau(3)<\tau(4), \ldots, \tau(2 m-1)<\tau(2 m) \\
\tau(1)<\tau(3)<\ldots<\tau(2 m-1), \\
\tau(2 m+1)<\tau(2 m+2)<\ldots<\tau(n)\rangle_{K} .
\end{gathered}
$$

Пусть $\mathbf{V}$ - некоторое многообразие алгебр Пуассона (все необходимые сведения о многообразиях PI-алгебр можно найти, например, в монографиях $[3,4]), \operatorname{Id}(\mathbf{V})-$ идеал тождеств многообразия $\mathbf{V}$. Обозначим:

$$
\begin{array}{cc}
P_{n}(\mathbf{V})=P_{n} /\left(P_{n} \cap I d(\mathbf{V})\right), & c_{n}(\mathbf{V})=\operatorname{dim} P_{n}(\mathbf{V}), \\
Q_{2 n}(\mathbf{V})=Q_{2 n} /\left(Q_{2 n} \cap I d(\mathbf{V})\right), & q_{2 n}(\mathbf{V})=\operatorname{dim} Q_{2 n}(\mathbf{V}), \\
R_{n}(\mathbf{V})=R_{n} /\left(R_{n} \cap I d(\mathbf{V})\right), & r_{n}(\mathbf{V})=\operatorname{dim} R_{n}(\mathbf{V}) .
\end{array}
$$

Лемма 1. Пусть $F=F(X), X=\left\{x_{1}, x_{2}, \ldots\right\}$, основное поле произвольно и элементь

$$
u_{s}^{2 n}\left(x_{1}, \ldots, x_{2 n}\right), \quad s=1, \ldots, q_{2 n}(F),
$$

образуют базис пространства $Q_{2 n}, n>0$. Тогда полилинейные элементы от переменных $x_{1}, \ldots, x_{n}$ вида

$$
\begin{gathered}
x_{1} \cdot \ldots \cdot x_{n} \\
x_{i_{1}} \cdot \ldots \cdot x_{i_{n-2 k}} \cdot u_{s}^{2 k}\left(x_{j_{1}}, \ldots, x_{j_{2 k}}\right), \\
2 \leq 2 k \leq n, \quad s=1, \ldots, q_{2 k}(F), \quad i_{1}<\ldots<i_{n-2 k}, \quad j_{1}<\ldots<j_{2 k},
\end{gathered}
$$

будут образовывать базис пространства $R_{n}$.

Доказательство. Очевидно, что любой элемент из $R_{n}$ является линейной комбинацией элементов вида (2).

Покажем, что элементы вида (2) линейно независимы в $R_{n}$. Предположим противное. Пусть выполнено нетривиальное линейное соотношение:

$$
\sum_{\substack{0 \leq 2 k \leq n \\ 1 \leq j_{1}<\ldots<j_{2 k} \leq n}} \alpha_{j_{1} \ldots j_{2 k}}^{k, s} x_{i_{1}} \cdot \ldots \cdot x_{i_{n-2 k}} \cdot u_{s}^{2 k}\left(x_{j_{1}}, \ldots, x_{j_{2 k}}\right)=0 .
$$

Выберем такое минимальное значение $k$, при котором $\alpha_{j_{1} \ldots j_{2 k}}^{k, s} \neq 0$. Подставим в этом случае вместо переменных $x_{i_{1}}, \ldots, x_{i_{n-2 k}}$ единицу. Тогда из (3) будет следовать такое нетривиальное линейное соотношение:

$$
\sum_{s=1}^{q_{2 k}(F)} \beta_{s} u_{s}^{2 k}\left(x_{j_{1}}, \ldots, x_{j_{2 k}}\right)=0
$$

где не все $\beta_{s}$ равны 0, что противоречит линейной независимости элементов (1).

Лемма 2. Пусть V - некоторое многообразие алгебр Пуассона над произвольным полем. Тогда 
(i) полилинейнье элементы

$$
u_{s}^{2 n}\left(x_{1}, \ldots, x_{2 n}\right), \quad s=1, \ldots, q_{2 n}(\mathbf{V}),
$$

образуют базис пространства $Q_{2 n}(\mathbf{V})$ тогда и только тогда, когда полилинейные элементы от переменных $x_{1}, \ldots, x_{n}$ вида

$$
\begin{gathered}
x_{1} \cdot \ldots \cdot x_{n}, \\
x_{i_{1}} \cdot \ldots \cdot x_{i_{n-2 k}} \cdot u_{s}^{2 k}\left(x_{j_{1}}, \ldots, x_{j_{2 k}}\right), \\
2 \leq 2 k \leq n, \quad s=1, \ldots, q_{2 k}(\mathbf{V}), \quad i_{1}<\ldots<i_{n-2 k}, \quad j_{1}<\ldots<j_{2 k},
\end{gathered}
$$

образуют базис пространства $R_{n}(\mathbf{V})$.

(ii) для любого натурального числа п выполнено равенство

$$
r_{n}(\mathbf{V})=1+\sum_{2 \leq 2 k \leq n} C_{n}^{2 k} q_{2 k}(\mathbf{V})
$$

где $C_{n}^{2 k}-$ число сочетаний из $n$ по $2 k$.

Доказательство. $(i)$ Доказательство линейной независимости элементов вида (5) аналогично доказательству линейной независимости элементов вида (2) в лемме 1. Поэтому остается показать, что любой элемент из $R_{n}(\mathbf{V})$ линейно выражается через элементы вида (5). Пусть $f\left(x_{1}, \ldots, x_{n}\right) \in R_{n}(\mathbf{V})$.

Дополним элементы (4) до базиса пространства $Q_{2 k}, 0 \leq 2 k \leq n$ :

$$
u_{s}^{2 k}\left(x_{1}, \ldots, x_{2 k}\right), v_{t}^{2 k}\left(x_{1}, \ldots, x_{2 k}\right), s=1, \ldots, q_{2 k}(\mathbf{V}), t=1, \ldots, q_{2 k}(F)-q_{2 k}(\mathbf{V}) .
$$

Тогда из леммы 1 следует, что элемент $f\left(x_{1}, \ldots, x_{n}\right)$ представим в виде следующей линейной комбинации:

$$
\begin{gathered}
f\left(x_{1}, \ldots, x_{n}\right)=\sum_{\substack{0 \leq 2 k \leq n, 1 \leq s \leq q_{2 k}(\mathbf{V}), 1 \leq j_{1}<\ldots<j_{2 k} \leq n}} \alpha_{j_{1} \ldots j_{2 k}}^{k, s} x_{i_{1}} \cdot \ldots \cdot x_{i_{n-2 k}} \cdot u_{s}^{2 k}\left(x_{j_{1}}, \ldots, x_{j_{2 k}}\right)+ \\
+\sum_{\substack{0 \leq 2 k \leq n, 1 \leq t \leq q_{2 k}(F)-q_{2 k}(\mathbf{V}), 1 \leq j_{1}<\ldots<j_{2 k} \leq n}} \beta_{j_{1} \ldots j_{2 k}}^{k, t} x_{i_{1}} \cdot \ldots \cdot x_{i_{n-2 k}} \cdot v_{t}^{2 k}\left(x_{j_{1}}, \ldots, x_{j_{2 k}}\right) .
\end{gathered}
$$

Предположим, что в равенстве (6) хотя бы один из элементов $\beta_{j_{1} \ldots j_{2 k}}^{k, t}$ не равен нулю. Выберем такое минимальное значение $k$, при котором $\beta_{j_{1} \ldots j_{2 k}}^{k, t} \neq 0$. Подставим вместо переменных $x_{i_{1}}, \ldots, x_{i_{n-2 k}}$ единицу. Получим такое равенство:

$$
f\left(1, \ldots, x_{j_{1}}, \ldots, x_{j_{2 k}}, \ldots, 1\right)=\sum_{s=1}^{q_{2 k}(\mathbf{V})} \epsilon_{s} u_{s}^{2 k}\left(x_{j_{1}}, \ldots, x_{j_{2 k}}\right)+\sum_{t=1}^{q_{2 k}(F)-q_{2 k}(\mathbf{V})} \delta_{t} v_{t}^{2 k}\left(x_{j_{1}}, \ldots, x_{j_{2 k}}\right),
$$

где не все $\delta_{t}$ равны 0. Так как левая часть равенства $(7)$ принадлежит $Q_{2 k}(\mathbf{V})$ и не все $\delta_{t}$ равны 0 , то элементы вида (4) не являются базисом пространства $Q_{2 k}(\mathbf{V})$. Противоречие.

Пункт $($ ii) следует из пункта $(i)$.

Теорема 2. Пусть V - нетривиальное многообразие алгебр Пуассона над произвольньм полем. тогда либо либо

1) $r_{n}(\mathbf{V}) \geq 2^{n-1}$ для любого $n$,

2) найдется такой многочлен $a_{2 N} x^{2 N}+\ldots+a_{1} x+a_{0}$ степени $2 N \geq 0$ из кольца $\mathbb{Q}[x]$, ито для любого $n \geq 2 N$ будет выполнено равенство

$$
r_{n}(\mathbf{V})=a_{2 N} n^{2 N}+\ldots+a_{1} n+a_{0}, \quad a_{2 N} \neq 0
$$

причем либо либо

2a) $r_{n}(\mathbf{V}) \geq 1+\frac{n(n-1)}{2}, n \geq 1, u \frac{1}{(2 N) !} \leq a_{2 N} \leq \frac{1}{N ! 2^{N}}$,

2b) $r_{n}(\mathbf{V})=1$ для любого $n$ 
Доказательство. Пусть последовательность $\left\{r_{n}(\mathbf{V})\right\}_{n \geq 1}$ не ограничена полиномом. Тогда из предложения 5 работы [5] следует, что для любого целого положительного $n$ выполнено неравенство $q_{2 n}(\mathbf{V})>0$. С учетом леммы 2 , получаем:

$$
r_{n}(\mathbf{V})=1+\sum_{2 \leq 2 k \leq n} C_{n}^{2 k} q_{2 k}(\mathbf{V}) \geq 1+\sum_{2 \leq 2 k \leq n} C_{n}^{2 k}=2^{n-1}
$$

Пусть теперь последовательность $\left\{r_{n}(\mathbf{V})\right\}_{n \geq 1}$ ограничена полиномом. Пусть $N-$ максимальное число, при котором $q_{2 N}(\mathbf{V})>0$. Тогда из леммы 2 следует, что для любого $n \geq 2 N$ будет выполнено равенство

$$
r_{n}(\mathbf{V})=1+\sum_{k=1}^{N} C_{n}^{2 k} q_{2 k}(\mathbf{V}),
$$

т. е.найдется такой многочлен степени $2 N \geq 0$ с рациональными коэффициентами, что для любого $n \geq 2 N$

$$
r_{n}(\mathbf{V})=a_{2 N} n^{2 N}+\ldots+a_{1} n+a_{0}, \quad a_{2 N} \neq 0
$$

Пусть $N>0$. Так как $q_{2 n}(F)=\frac{(2 n) !}{n ! 2^{n}}$ для любого $n$ [6], то для любого $n \geq 2 N$ будет выполнено двойное неравенство:

$$
\sum_{k=0}^{N} C_{n}^{2 k} \leq r_{n}(\mathbf{V}) \leq \sum_{k=0}^{N} C_{n}^{2 k} \cdot \frac{(2 k) !}{k ! 2^{k}}
$$

Поэтому

$$
\frac{1}{(2 N) !} \leq a_{2 N} \leq \frac{1}{N ! 2^{N}}
$$

При этом заметим, что

$$
r_{n}(\mathbf{V}) \geq 1+C_{n}^{2} \cdot q_{2}(\mathbf{V}) \geq 1+\frac{n(n-1)}{2} .
$$

Если $N=0$, то $r_{n}(\mathbf{V})=1$ для любого $n$.

Пусть $\Lambda_{2 n}$ - алгебра Грассмана с единицей, $2 n$ образующими элементами $\left\{e_{1}, \ldots, e_{2 n}\right\}$ и операцией умножения «^». Введем в алгебре $\Lambda_{2 n}$ два новых умножения:

$$
a \cdot b=\frac{1}{2}(a \wedge b+b \wedge a), \quad\{a, b\}=a \wedge b-b \wedge a, \quad a, b \in \Lambda_{2 n} .
$$

Обозначим полученную алгебру Пуассона $\left(\Lambda_{2 n},+, \cdot,\{\},\right)$ через $G_{2 n}$.

Лемма 3 [5]. Пусть $N-$ произвольное натуральное число. Для алгебрь Пуассона $G_{2 N}$ над полем нулевой характеристики верны следующие утверждения:

(i) полилинейные тождества

$$
\{x, y, z\}=0, \quad\left\{x_{1}, y_{1}\right\} \cdot \ldots \cdot\left\{x_{N+1}, y_{N+1}\right\}=0
$$

порождают идеал тождеств алгебры $G_{2 N}$;

(ii) последовательность $\left\{r_{n}\left(G_{2 N}\right)\right\}_{n \geq 1}$ достигает нижней границь в неравенстве (8):

$$
c_{n}\left(G_{2 N}\right)=r_{n}\left(G_{2 N}\right)=\sum_{k=0}^{N} C_{n}^{2 k}, \quad n \geq 2 N,
$$

при этом $a_{2 N}=\frac{1}{(2 N) !}$.

Следующая лемма, в частности, показывает, что многообразие, порожденное алгеброй $G_{2}$, является наименьшим многообразием алгебр Пуассона в классе всех многообразий алгебр Пуассона, имеющих рост не ниже полиномиального.

Лемма 4 [5]. Для алгебры $G_{2}$ над полем нулевой характеристики вернь следующие утверждения:

(i) тождества

$$
\left\{x_{1}, x_{2}, x_{3}\right\}=0, \quad\left\{x_{1}, x_{2}\right\} \cdot\left\{x_{3}, x_{4}\right\}=0
$$

порождают идеал тождеств алгебры $G_{2}$; 
(ii) $q_{2}\left(G_{2}\right)=1, q_{2 n}\left(G_{2}\right)=0, n \geq 2$,

$$
c_{n}\left(G_{2}\right)=r_{n}\left(G_{2}\right)=1+\frac{n(n-1)}{2}, \quad n \geq 1 ;
$$

(iii) многообразие $\operatorname{var}\left(G_{2}\right)$ является наименьшим многообразием среди всех многообразий алгебр Пуассона $\mathbf{V}$, у которых последовательность $\left\{r_{n}(\mathbf{V})\right\}_{n \geq 1}\left(\left\{c_{n}(\mathbf{V})\right\}_{n \geq 1}\right)$ растет не ниже полинома, т.е.если для некоторого многообразия $\mathbf{V}$ последовательность $\left\{r_{n}(\mathbf{V})\right\}_{n \geq 1}\left(\left\{c_{n}(\mathbf{V})\right\}_{n \geq 1}\right)$ растет не ниже полинома, то $G_{2} \in \mathbf{V}$.

Заметим, что существует бесконечно много попарно различных многообразий алгебр Пуассона $\mathbf{V}$, у которых последовательность $\left\{r_{n}(\mathbf{V})\right\}_{n \geq 1}$ достигает нижней границы полиномиального роста. Пусть $S U_{N}=S U_{N}(K)$ - алгебра строго верхнетреугольных матриц порядка $N$ над полем $K$ и операцией умножения $\wedge$. В векторном пространстве $S U_{N} \oplus K$ над полем $K$ определим две операции умножения · и $\{$,$\} следующим образом:$

$$
\begin{gathered}
(a+\alpha) \cdot(b+\beta)=(\beta a+\alpha b)+\alpha \beta, \\
\{a+\alpha, b+\beta\}=[a, b],
\end{gathered}
$$

где $[a, b]=a \wedge b-b \wedge a, a, b \in S U_{N}, \alpha, \beta \in K$. Полученную алгебру Пуассона $\left(S U_{N} \oplus K, \cdot\{\}, K,\right)$ обозначим через $P S U_{N}$.

Лемма 5 [7]. Для алгебры Пуассона $P S U_{N}$ над полем нулевой характеристики верны следующие утверждения:

(i) полилинейные тождества

$$
\left\{x_{1}, x_{2}\right\} \cdot\left\{x_{3}, x_{4}\right\}=0, \quad\left\{x_{1}, x_{2}, \ldots, x_{N}\right\}=0
$$

порождают идеал тождеств алгебры $P S U_{N}$;

(ii) $q_{2}\left(P S U_{N}\right)=1, q_{2 n}\left(P S U_{N}\right)=0, n>1$,

$$
\begin{gathered}
r_{n}\left(P S U_{N}\right)=1+\frac{n(n-1)}{2}, \quad n \geq 1, \\
c_{n}\left(P S U_{N}\right)=1+\sum_{k=2}^{\min \{n, N-1\}} C_{n}^{k} \cdot(k-1) !, \quad n \geq 1 .
\end{gathered}
$$

\section{Библиографический список}

1. Farkas D. R. Poisson polynomial identities // Comm. Algebra. 1998. Vol. 26, № 2. P. 401-416.

2. Farkas D. R. Poisson polynomial identities. II // Arch. Math. (Basel). 1999. Vol. 72, № 4. P. 252-260.

3. Бахтурин Ю. А. Тождества в алгебрах Ли. М. : Наука, 1985.

4. Giambruno A., Zaicev M. V. Polynomial Identities and Asymptotic Methods. Math. Surv. and Monographs. Providence, R.I. : American Math. Soc., 2005. Vol. 122.

5. Рацеев С. М. Алгебры Пуассона полиномиального роста // Сиб. мат. журн. 2013. Т. 54, № 3. С. 700-711.

6. Mishchenko S. P., Petrogradsky V. M., Regev A. Poisson PI algebras // Trans. Amer. Math. Soc. 2007. Vol. 359, № 10. P. 4669-4694.

7. Череватенко О. И. О лиево нильпотентных алгебрах Пуассона // Научные ведомости БелГУ. Сер. Математика, физика. 2012. № 23(142), вып. 29. С. 14-16.

\section{On Poisson Customary Polynomial Identities}

\section{S. M. Ratseev}

Ulyanovsk State University, 42, Leo Tolstoy str., 432017, Ulyanovsk, Russia, RatseevSM@mail.ru

We study Poisson customary and Poisson extended customary polynomials. We show that the sequence of codimensions $\left\{r_{n}(V)\right\}_{n \geq 1}$ of every extended customary space of variety $\mathrm{V}$ of Poisson algebras over an arbitrary field is either bounded by a polynomial or at least exponential. Furthermore, if this sequence is bounded by polynomial then there is a polynomial $R(x)$ with rational coefficients such that $r_{n}(V)=R(n)$ for all sufficiently large $\mathrm{n}$. We present lower and upper bounds for the polynomials $\mathrm{R}(\mathrm{x})$ of an arbitrary fixed degree.

Key words: Poisson algebra, variety of algebras, growth of a variety. 


\title{
References
}

1. Farkas D. R. Poisson polynomial identities. Comm. Algebra, 1998, vol. 26, no. 2, pp. 401-416.

2. Farkas D. R. Poisson polynomial identities. II. Arch. Math. (Basel), 1999, vol. 72, no. 4, pp. 252-260.

3. Bahturin Yu. A. Identical relations in Lie algebras. Utrecht, VNU Sci. Press, 1987. 309 p. (Rus. ed. : Bahturin Yu. A. Tozhdestva v algebrah Li. Moscow, Nauka, 1985).

4. Giambruno A., Zaicev M. V. Polynomial Identities and Asymptotic Methods. Math. Surv. and Monographs.

Providence, R.I., American Math. Soc., 2005, vol. 122. 5. Ratseev S. M. Poisson algebras of polynomial growth. Siberian Math. J. 2013, vol. 54, no. 3, pp. 555-565.

6. Mishchenko S. P., Petrogradsky V. M., Regev A. Poisson PI algebras. Trans. Amer. Math. Soc., 2007, vol. 359, no. 10 , pp. 4669-4694.

7. Cherevatenko O. I. On nilpotent Leibnitz algebras. Nauchnyye vedomosti BelGU. Ser. Matematika. Fizika [Belgorod State University Scientific Bulletin. Ser. Mathematics. Physics], 2012, no. 23(142), iss. 29, pp. 14-16.

УДК 517.54

\section{НОВЫЙ ПОДХОД К РЕШЕНИЮ КРАЕВОЙ ЗАДАЧИ РИМАНА С БЕСКОНЕЧНЫМ ИНДЕКСОМ}

\section{Р. Б. Салимов ${ }^{1}$, Э. Н. Карабашева ${ }^{2}$}

\begin{abstract}
${ }^{1}$ Доктор фризико-мататематических наук, профрессор, заведующий кафедрой высшей математики, Казанский государственный архитектурно-строительный университет, salimov@5354.ru

${ }^{2}$ Аспирант касредры высшей математики, Казанский государственный архитектурно-строительный университет, enkarabasheva@bk.ru

В работе рассматривается краевая задача Римана с бесконечным индексом, когда краевое условие задачи задается на действительной оси комплексной плоскости. Для решения этой задачи используется подход, основанный на устранении бесконечного разрыва аргумента коэсрфициента краевого условия и аналогичный тому, с помощью которого в случае конечного индекса задачи ранее в работах Ф. Д. Гахова устранялись разрывы коэффрициента краевого условия с помощью специально подобранных фрункций, отличных от используемых в настоящей работе.
\end{abstract}

Ключевые слова: краевая задача Римана, аналитическая фрункция, бесконечный индекс.

\section{1. ВВЕДЕНИЕ. ПОСТАНОВКА ЗАДАЧИ}

Пусть $D^{+}$и $D^{-}-$соответственно верхняя и нижняя полуплоскости в плоскости переменного $z=x+i y$ с действительной осью $L, \Phi^{+}(z)$ и $\Phi^{-}(z)-$ функции, аналитические соответственно в областях $D^{+}$и $D^{-}$. Требуется определить функции $\Phi^{+}(z)$ и $\Phi^{-}(z)$, ограниченные в областях $D^{+}$и $D^{-}$соответственно, если их граничные значения удовлетворяют условию

$$
\Phi^{+}(t)=G(t) \Phi^{-}(t)+g(t), t \in L,
$$

в котором $G(t), g(t)$ - заданные на $L$ функции. В случае, когда $\ln G(t)$ и $g(t)-$ функции, удовлетворяющие условию $H_{L}$ (условию Гёльдера) всюду на $L$, включая окрестность точки $t=\infty$ [1, с. 67], решение задачи (1) дано в монографиях $[1$, с. 136-139; 2, с. 118-121]. Решение задачи зависит от её индекса, равного $(\arg G(+\infty)-\arg G(-\infty)) / 2 \pi$.

Начало исследованиям задачи (1) в случае, когда ее индекс бесконечен, т.е. $\arg G(+\infty)-$ $-\arg G(-\infty)=\infty$, было положено Н. В. Говоровым. Результаты его работ в дальнейшем вошли в монографию [3]. Этой проблеме посвящен ряд работ других авторов; отметим из них статьи [4-7], в которых изучены новые случаи задачи Римана с бесконечным индексом, в статье [8] рассмотрен особый случай задачи, в [9] изучен случай, когда в задаче (1) при $g(t) \equiv 0$ в качестве $L$ берется произвольный гладкий замкнутый контур, в окрестностях некоторых точек которого $\arg G(t)$ неограничен.

Авторы указанного ряда работ решение задачи (1) получают путем построения канонического решения - частного решения соответствующей однородной задачи:

$$
\Phi^{+}(t)=G(t) \Phi^{-}(t), \quad t \in L,
$$

обладающего нужными свойствами, аналогично тому, как это было сделано ранее Н. В. Говоровым. 